\title{
REVIEW
}

\section{Cholesterol lowering and coronary artery disease: mechanisms of risk reduction}

\author{
R A Archbold, A D Timmis
}

Hypercholesterolaemia has long been recognised as one of the major reversible risk factors for coronary artery disease. Among 356222 men screened for the multiple risk factor intervention trial (MRFIT), there was a close linear relation between serum cholesterol and mortality during a six year follow up period. ${ }^{1}$ The relation extended right across the range of serum cholesterol measurements, indicating that even within the so called "normal" range, individuals who have a lower serum cholesterol have a lower risk of cardiovascular death. Nevertheless, it was not until the publication of the Scandinavian simvastatin survival study (4S) and the cholesterol and recurrent events (CARE) trial that treatment of hypercholesterolaemia in patients with coronary artery disease was shown to produce corresponding reductions in long term cardiovascular and all cause mortality. ${ }^{23}$ An important observation in these studies was that the survival curves separated within two years, indicating the benefits of treatment occurred very early. Any mechanistic theory accounting for these benefits must, therefore, be able to account for this early response to treatment. Three main mechanisms have been suggested: (a) modification of the progression of coronary atheroma; (b) stabilisation of the coronary plaque, reducing the risk of rupture; (c) correction of coronary endothelial function with restoration of normal vasomotor responses.

\section{Modification of the progression of coronary artery disease}

Current theories of atherogenesis consider oxidative modification of low density lipoprotein (LDL) as the central event that initiates and propagates coronary artery disease. ${ }^{4}$ Properties of oxidised LDL which contribute to its atherogenic potential are its chemotaxis for circulating monocytes, inhibition of tissue macrophage migration away from the arterial intima, enhanced macrophage uptake of LDL by scavenger receptors to form foam cells, and its cytotoxicity, causing endothelial cell injury. ${ }^{5}$ It was a logical expectation, therefore, that lowering LDL cholesterol with specific treatment would slow the progression of coronary disease and perhaps also induce regression. This hypothesis has been tested in a large number of coronary angiographic studies which have ran- domised patients to lipid lowering interventions (diet, exercise, partial ileal bypass, and/or drugs) or control (diet or "usual care"). The results of 10 studies were reviewed by Brown et $a l .{ }^{6}$ LDL cholesterol reductions of about $35 \%$ were achieved in the intervention groups and these were associated with a favourable shift in the balance between progression and regression of coronary artery disease. It is tempting to conclude, therefore, that the beneficial effects of treating hypercholesterolaemia in patients with coronary artery disease are mediated by a direct effect on the atherogenic process which leads to reduction of coronary luminal stenosis.

A closer analysis of the data, however, indicates that this conclusion is unlikely to be correct. ${ }^{67}$ Firstly, it is now well established that the angiographic severity of disease correlates poorly with coronary flow and severity of symptoms. Moreover, there is no apparent relation between the severity of lesions and their propensity to cause future cardiac events. ${ }^{89}$ In addition, the average reduction in coronary stenosis associated with cholesterol reduction in the angiographic trials was no more than $1-2 \%$ or $0.05 \mathrm{~mm}$, a very small change that is disproportionate to the marked reduction in adverse clinical events seen in the $4 \mathrm{~S}$ and CARE studies. It is unlikely, therefore, that the benefits of cholesterol lowering in secondary prevention trials can be explained by changes in the severity of coronary stenosis. This is not to say that these changes may not contribute to the long term benefits of treatment but they are unlikely to be the major determinant of benefit.

\section{Plaque stabilisation}

Pathological studies have confirmed that acute ischaemic events in patients with coronary artery disease are usually provoked by rupture of an atheromatous plaque. ${ }^{10}{ }^{11}$ This exposes plaque contents to the circulation and provides a potent thrombogenic stimulus. The severity of the thrombotic response determines the clinical presentation. Thus, minor plaque disruption and thrombosis may be asymptomatic. ${ }^{12}$ Indeed, it is now thought that subclinical episodes of plaque disruption with subsequent healing is a major mechanism in the progression of coronary artery disease, with 
seven out of 10 high grade stenoses showing evidence of healed disruption within the plaque. ${ }^{13}$ More severe events may lead to subtotal or total thrombotic coronary occlusion presenting as unstable angina or myocardial infarction, respectively. ${ }^{14}$

Atheromatous plaques comprise a cholesterol core encapsulated by a fibrous tissue "cap". The cap is made up of an extracellular matrix of structural proteins (collagen, elastin, proteoglycans) synthesised by vascular smooth muscle cells. Macrophages and T lymphocytes predominate in an inflammatory cell infiltrate. Interestingly, the vulnerability of the plaque to rupture is determined not by the size of the plaque, but by the interaction of circumferential stress within the arterial wall, the cholesterol content of the plaque, and the strength of the cap. ${ }^{15}$ Circumferential stress is determined largely by systolic blood pressure ${ }^{16}$ and its distribution across a lipid laden plaque is maximal near the vulnerable shoulder region. ${ }^{15}$ Postmortem studies have revealed that plaques in which the lipid pool exceeds $40 \%$ of the plaque volume are particularly prone to rupture. The strength of the cap is related directly to its smooth muscle and collagen concentration, and inversely to its macrophage content. ${ }^{17}$

The separation of the circulation from the contents of the plaque is ultimately dependent upon the integrity of the fibrous cap. Concepts are evolving which might explain how cap strength is determined at a molecular level. It is now clear that the cap is a dynamic structure in which there is a constant turnover of matrix material. ${ }^{18}$ It is thought that the balance between collagen synthesis and degradation plays an important role in determining cap strength and, hence, vulnerability to rupture. Connective tissue proteins are degraded by protease enzymes, the most important of which are considered to be the matrix metalloproteinases (MMPs). ${ }^{19}$ They are secreted as inactive precursors by a range of cells, including macrophages, and are inhibited by tissue inhibitors of metalloproteinases (TIMPs). An excess of active enzymes over precursors and inhibitors has been demonstrated in the vulnerable shoulder region of human plaques. ${ }^{20}$ Furthermore, macrophages and $\mathrm{T}$ lymphocytes that accumulate at the site of rupture in vulnerable plaques secrete inflammatory mediators such as interferon $\gamma$, tumour necrosis factor, and interleukin 1, which inhibit collagen synthesis by smooth muscle cells. ${ }^{18}{ }^{21}$ Together, these processes are thought to contribute to weakening of the fibrous cap.

It has been hypothesised that treatments which lower blood cholesterol might increase plaque stability, so reducing the risk of ischaemic events. A reduction in the lipid pool within the plaque was initially thought the most likely mechanism of increased stability. However, animal models suggest that cholesterol lowering can alter plaque structure, not only by reducing the lipid pool, but also by increasing the smooth muscle collagen concentration and by reducing the inflammatory infiltrate..$^{22-24}$ Intriguingly, it has recently been shown that a lipid lowering diet in a rabbit model of athero- sclerosis produced a progressive reduction in macrophage number and expression of MMP-1 (interstitial collagenase) within plaques. Proteolytic activity caused by a variety of MMPs was also decreased, while interstitial collagen increased in the lipid lowering group. ${ }^{25}$ It follows that one of the mechanisms by which lipid lowering therapy might reduce cardiac events is by favourably altering the balance between collagen synthesis and degradation within the fibrous cap.

The hypothesis that lowering blood cholesterol and its LDL fraction favourably influences determinants of plaque stability is attractive. It provides not only a plausible mechanism whereby treatment of hypercholesterolaemia in patients with coronary artery disease might reduce susceptibility to myocardial infarction and death, but also for the reduced progression of disease in the randomised angiographic trials.

\section{Endothelial function}

The vascular endothelium has been the focus of considerable interest. ${ }^{26}$ Among its numerous regulatory functions, control of arterial tone is probably the best characterised. The endothelium secretes a range of vasoactive compounds including endothelin and angiotensin II (vasoconstrictors), and nitric oxide and prostacyclin (vasodilators). ${ }^{27}$ Acetylcholine is a potent stimulus for nitric oxide secretion by the vascular endothelium ${ }^{28}$ and has been used experimentally as a tool for investigating endothelial function. Thus, in a variety of animal models, acetylcholine injection provokes endothelium dependent vasodilatation through nitric oxide release. In animal models of atherosclerosis, however, acetylcholine elicits either an attenuated vasodilator response or, paradoxically, a vasoconstrictor response, ${ }^{29}$ which is thought to be consequent to loss of nitric oxide function and the unopposed endothelial independent vasoconstrictor action of acetylcholine. It has also been shown that endothelial dysfunction is present in hypercholesterolaemic animals in the absence of atherosclerosis. ${ }^{30}$ Similarly, in human studies it has been demonstrated that endothelium dependent vasodilator responses are impaired in patients with atherosclerosis ${ }^{31} 32$ and in patients with hypercholesterolaemia but angiographically normal coronary arteries..$^{32}$ In human volunteers with blood cholesterol levels towards the upper end of the "normal range", endothelium dependent vasodilator responses to methacholine chloride (a muscarinic agonist) are impaired compared with those with a lower blood cholesterol. ${ }^{34}$ Clearly, therefore, the potential for hypercholesterolaemia to modify arterial endothelial function adversely can be demonstrated throughout the range of normal cholesterol values.

It has been suggested the endothelial dysfunction that characterises coronary artery disease plays a major role, not only in the pathogenesis of the disorder but also in its ischaemic manifestations, the loss of normal vasodilator function tending to intensify the myocardial perfusion deficit. ${ }^{35}$ Evidence is 
accumulating that the endothelial dysfunction is often reversible by cholesterol lowering. Anderson and colleagues showed that, in patients with coronary artery disease and a mean blood cholesterol concentration of $5.4 \mathrm{mmol} / 1$, treatment with cholesterol lowering drugs over a 12 month period produced substantial improvement of coronary endothelial function as reflected by coronary vasomotor responses to acetylcholine infusion. ${ }^{36}$ Leung et al reported improvement in acetylcholine induced coronary dilatation in hypercholesterolaemic patients with angiographically normal coronary arteries after six months of lipid lowering treatment, ${ }^{37}$ while further reports suggest that statin therapy can improve endothelial function in hypercholesterolaemic subjects within a few weeks. ${ }^{38}{ }^{39}$ Recent data suggest that this improvement is mediated by an increased bioavailability of nitric oxide. ${ }^{40}$ The ability of cholesterol reduction to improve coronary vasomotor responses is clearly of potential benefit and, moreover, occurs very early after initiation of treatment within a time frame that would be compatible with the early clinical benefits of statins in the $4 S$ and CARE trials.

Demonstration of improved coronary endothelial responses to lipid lowering drugs falls short of confirming that treatment of this type can reduce ischaemic events in patients with coronary artery disease. Controlled trials have investigated this relation by studying the effect of statin therapy on Holter ST changes in patients with coronary artery disease. Seven hundred and sixty eight patients out of 885 in the regression growth evaluation statin study (REGRESS) underwent ST monitoring before and up to two years after randomisation to treatment with pravastatin. A number of measures of ischaemia were significantly reduced in the treatment group. ${ }^{41}$ Andrews et al examined the effects of cholesterol reduction with lovastatin on Holter ST changes in patients with coronary artery disease. ${ }^{42}$ After six months of treatment, significant reductions in ischaemic episodes were demonstrated in patients treated with lovastatin, but in patients treated with placebo ischaemic episodes were unaffected. It is possible to infer from these data that patients with coronary artery disease treated with statins exhibit a very rapid reduction in ischaemia, which occurs too early to be attributable to alterations in the progression of coronary disease. This reduction in ischaemic burden is best accounted for by improvement in coronary endothelial dilator responsiveness.

\section{Additional mechanisms}

It is clear that lipid lowering by statin therapy reduces the risk of cardiovascular events and death, and we have outlined potential mechanisms which may account for these benefits. It is less clear whether this decrease in events is attributable solely to the reduction in cholesterol achieved, or whether specific agents may have additional properties independent of cholesterol lowering. Cardiac events have been reduced by lipid lowering interventions other than statins, ${ }^{43-45}$ but fewer events and smaller populations make comparisons with the statin trials difficult. Trials of clinical outcome powered to compare lipid lowering interventions in groups which achieve similar plasma cholesterol concentrations are impractical and, perhaps, unwarranted given the comparable reductions in adverse clinical events with different statins in the $4 \mathrm{~S}$ and CARE studies. Nevertheless, it is interesting that Libby's group has shown that a similar degree of lipid lowering by pravastatin to that achieved by diet in rabbits produced better coronary vasodilator function and a greater reduction in macrophage infiltrate within atheromatous plaques. ${ }^{46}$ They concluded that pravastatin had beneficial effects independent of plasma lipoprotein concentrations.

\section{FIBRINOGEN AND PLATELET EFFECTS}

Pravastatin has been reported to lower plasma fibrinogen concentration which is unaffected by simvastatin, despite similar falls in cholesterol, ${ }^{47}$ while the increased platelet activity associated with hypercholesterolaemia may also be reduced by statin therapy. ${ }^{48}$ Studies of statin effects on fibrinogen concentration and platelet function, however, have not produced consistent results. ${ }^{49}$

ANTIOXIDANT PROPERTIES

Further studies have demonstrated that lipid lowering agents from several pharmacological classes reduce the susceptibility of LDL to oxidation, and it has been suggested that this is not entirely caused by LDL cholesterol reduction. ${ }^{50}$ In rabbits fed a high cholesterol diet for 12 weeks with or without lovastatin, blood markers of lipid peroxidation were lower and endogenous antioxidant activity was higher in the statin group, despite similar cholesterol concentrations. Lovastatin also reduced in vitro leucocyte induced LDL oxidation. ${ }^{51}$

ANTI-SMOOTH MUSCLE PROLIFERATION

The ability of statins to lower cholesterol is mediated by their inhibition of 3-hydroxy-3methylglutaryl coenzyme A reductase, the rate limiting enzyme in cholesterol synthesis. The synthesis of other compounds in the biosynthetic pathway distal to this enzyme, which are involved in the regulation of cell growth, is similarly inhibited. Hence, a number of statins (fluvastatin, simvastatin, lovastatin, but not pravastatin) have been shown to inhibit smooth muscle cell migration in vitro and smooth muscle cell proliferation in vivo. ${ }^{52}$ The implications of these antiproliferative properties is controversial and may, in fact, be deleterious since vascular smooth muscle cells are now thought to contribute importantly to plaque stability.

\section{Perspective}

Although the reductions in cardiac events and death in the landmark lipid lowering trials were considerable, event rates remained frustratingly high among those who received active treatment. The trials of cholesterol reduction randomised patients on the basis of total or LDL cholesterol concentrations. Other 
lipoproteins such as $\mathrm{Lp}(\mathrm{a})$ or high density lipoprotein (HDL), or triglycerides may be important particularly in those with "normal" LDL. Clearly, cholesterol reduction must be viewed in conjunction with the other risk factors for coronary artery disease, such as smoking, diabetes, and hypertension, which may still be operating. Indeed, smoking increases inflammatory cell adhesion to vascular endothelium, ${ }^{53}$ enhances LDL modification by endothelial cells, ${ }^{54}$ and impairs coronary vasodilator responses, ${ }^{55}$ directly opposing several mechanisms that may contribute to the beneficial effects of lipid lowering therapy.

Adjuvant therapy to lipid lowering may permit further reductions in event rates. It has been demonstrated, for example, that a combination of antioxidant and statin therapy conferred a greater improvement in coronary endothelial function than statin therapy alone in patients with coronary artery disease.$^{36}$ More recently, Galis et al reported that $\mathrm{N}$-acetylcysteine, a reactive oxygen species scavenger, reduced MMP-9 expression in plaques and on culture of lesion foam cells in a rabbit model of atherosclerosis. ${ }^{56}$ Recent interest has surrounded the potential role of infective agents, including Chlamydia pneumoniae, in the pathogenesis of coronary artery disease. Atherosclerosis is accelerated in rabbits infected with $C$ pneumoniae and treatment with azithromycin prevents it. ${ }^{57}$ Studies suggest that antichlamydial antibiotics may improve clinical outcome in human patients with coronary artery disease. ${ }^{58}$ An adjuvant role for macrolide antibiotics is, therefore, an exciting possibility.

\section{Conclusion}

There is unlikely to be a simple explanation for the beneficial effects of lipid lowering in reducing ischaemic events in patients with coronary artery disease. Changes in the progression of coronary disease may influence ischaemic events in the longer term, but cannot explain the rapid reduction of risk that was demonstrated in $4 \mathrm{~S}$ and CARE. Episodes of plaque rupture underlie most acute coronary syndromes, and cholesterol lowering alters plaque structure in a manner likely to increase stability. Cholesterol lowering also improves coronary vasomotor responses early after the initiation of treatment. The rapid reduction of risk observed in the lipid lowering trials is, therefore, most likely to be attributable to reductions in plaque vulnerability and improvements in coronary endothelial function.

1 Stamler J, Wentworth D, Neaton JD for the MRFIT Research Group. Is relationship between serum cholesterol and risk of premature death from coronary heart disease continuous and graded? Findings in 356,222 primary screenees of the multiple risk factor intervention tria (MRFIT). $\mathscr{\text { IAMA }}$ 1986;256:2823-8.

2 Scandinavian Simvastatin Survival Study Group. Randomised trial of cholesterol lowering in 4444 patients with coronary heart disease: the Scandanavian simvastatin survival study (4S). Lancet 1994;344:1383-9.

3 Sacks FM, Pfeffer MA, Moye LA, et al for the Cholesterol and Recurrent Events Trial Investigators. The effect of pravastatin on coronary events after myocardial infarction in patients with average cholesterol levels. $N$ Engl $f \mathrm{Med}$ in patients with

4 Yla-Herttuala S, Palinski W, Rosenfeld ME, et al. Evidence for the presence of oxidatively modified low density
lipoprotein in atherosclerotic lesions of rabbit and man. $\mathcal{F}$ Clin Invest 1989;84:1086-95.
5 Steinberg D, Parthasarathy S, Carew TE, et al. Beyond cholesterol: modifications of low-density-lipoprotein that increase

6 Brown BG, Zhao X-Q, Sacco DE, et al. Lipid lowering and plaque regression. New insights into prevention of plaque disruption and clinical events in coronary disease. Circulation 1993;87:1781-91

7 Vos J, de Feyter PJ, Simoons ML, et al. Retardation and arrest of progression or regression of coronary artery disease: a review. Prog Cardiovasc Dis 1993;35:435-54.

8 Ambrose J, Tannenbaum MA, Alexopoulos $\mathrm{D}$, et al. Angiographic progression of coronary artery disease and the development of myocardial infarction. $f \mathrm{Am}$ Coll Cardiol 1988;12:56-62.

9 Little WC, Constantinescu M, Applegate RJ, et al. Can coronary angiography predict the site of a subsequent myocardial infarction in patients with mild-to-moderate coronary artery disease? Circulation 1988;78:1157-66.

10 Falk E. Plaque rupture with severe pre-existing stenosis precipitating coronary thrombosis: characteristics of coronary atherosclerotic plaques underlying fatal occlusive thrombi. Br Heart F 1983;50:127-34.

11 Davies MJ, Thomas AC. Plaque fissuring - the cause of acute myocardial infarction, sudden ischaemic death, and crescendo angina. Br Heart $f$ 1985;53:363-73.

12 Davies MJ, Bland JM, Hangartner JRW, et al. Factors influencing the presence or absence of acute coronary artery thrombi in sudden ischaemic death. Eur Heart 7 1989;10: 203-8.

13 Davies MJ. Stability and instability: two faces of coronary atherosclerosis. The Paul Dudley White Lecture 1995. Circulation 1996;94:2013-20.

14 Fuster V, Badimon L, Badimon JJ, et al. The pathogenesis of coronary artery disease and the acute coronary syndromes (first of two parts). N Engl f Med 1992;326:242-50.

15 Richardson PD, Davies MJ, Born GVR. Influence of plaque configuration and stress distribution on fissuring of coronary atherosclerotic plaques. Lancet 1989;ii:941-4.

16 Lee RT, Kamm RD. Vascular mechanics for the cardiologist. F Am Coll Cardiol 1994;23:1289-95.

17 Davies MJ, Richardson PD, Woolf N, et al. Risk of thrombosis in human atherosclerotic plaques: role of extracellular ipid, macrophage, and smooth muscle content. Br Heart $\mathcal{F}$ 1993;69:377-81.

18 Libby P. Molecular bases of the acute coronary syndromes. Circulation 1995;91:2844-50.

19 Dollery CM, McEwan JR, Henney AM. Matrix metalloproteinases and cardiovascular disease. Circ Res 1995;77:863-

20 Galis ZS, Sukhova GK, Lark MW, et al. Increased expression of matrix metalloproteinases and matrix degrading activity in vulnerable regions of human atherosclerotic plaques. f Clin Invest 1994;94:2493-2503.

21 van der Wal AC, Becker AE, van der Loos CM, et al. Site of intimal rupture or erosion of thrombosed coronary atherosclerotic plaques is characterized by an inflammatory process irrespective of the dominant plaque morphology. Circulation 1994;89:36-44.

22 Small DM, Bond MG, Waugh D, et al. Physiochemical and histological changes in the arterial wall of non-human primates during progression and regression of atherosclerosis. f Clin Invest 1984;73:1590-1605.

23 Harrison DG, Armstrong ML, Freiman PC, et al. Restoration of endothelial-dependent relaxation by dietary treatment of atherosclerosis. F Clin Invest 1987;80:180811 .

24 Shiomi M, Ito T, Tsukada T, et al. Reduction of serum cholesterol levels alters lesional composition of atherosclerotic plaques. Effect of pravastatin sodium on atheroscerosis in mature WHHL rabbits. Arterioscler Thromb Biol 1995;15: mature W $1938-44$.

25 Aikawa M, Rabkin E, Okada Y, et al. Lipid lowering by diet reduces matrix metalloproteinase activity and increases collagen content of rabbit atheroma: a potential mechanism of lesion stabilisation. Circulation 1998;97:2433-44.

26 Celermajer DS. Endothelial dysfunction: does it matter? Is it reversible? F Am Coll Cardiol 1997;30:325-33.

27 Luscher TF, Barton M. Biology of the endothelium. Clin Cardiol 1997;20(suppl II):II3-10.

28 Furchgott RF, Zawadzki JV. The obligatory role of the endothelial cells in the relaxation of arterial smooth muscle by acetylcholine. Nature 1980;288:373-6.

29 Freiman PC, Mitchell GG, Heistad DD, et al. Atherosclerosis impairs endothelium-dependent vascular relaxation to acetylcholine and thrombin in primates. Circ Res 1986;58: 783-9.

30 Chappell SP, Lewis MJ, Henderson AH. Effect of lipid lowering on endothelium dependent relaxation in rabbit aortic preparations. Cardiovasc Res 1987;21:34-8.

31 Ludmer PL, Selwyn AP, Shook TL, et al. Paradoxical vasoconstriction induced by acetylcholine in atherosclerotic coronary arteries. N Engl F Med 1986;315:1046-51.

32 Zeiher AM, Drexler H, Wollschlager $\mathrm{H}$, et al. Modulation of coronary vasomotor tone in humans. Progressive endotheial dysfunction with different early stages of coronary atherosclerosis. Circulation 1991;83:391-401.

33 Vita JA, Treasure CB, Nabel EG, et al. Coronary vasomotor response to acetylcholine relates to risk factors for coronary artery disease. Circulation 1990;81:491-7.

34 Steinberg HO, Bayazeed B, Hook G, et al. Endothelial dysfunction is associated with cholesterol levels in the high normal range in humans. Circulation 1997;96:3255-7. 
35 Meredith IT, Yeung AC, Weidinger FF, et al. Role of impaired endothelium-dependent vasodilation in ischemic manifestations of corona artery disease. Circulation 1993, 87(suppl V):V56-66.

36 Anderson TJ, Meredith IT, Yeung AC, et al. The effect of cholesterol-lowering and antioxidant therapy on endothelium-dependent coronary vasomotion. $N$ Engl $f$ Med 1995;332:488-93.

37 Leung W-H, Lau C-P, Wong C-K. Beneficial effect of cholesterol-lowering therapy on coronary endotheliumdependent relaxation in hypercholesterolaemic patients. Lancet 1993;341:1496-500.

38 O'Driscoll G, Green D, Taylor R. Simvastatin, an HMG-coenzyme A reductase inhibitor improves endothelial function within one month. Circulation 1997:95:112631.

39 Schmieder RE, Schobel HP. Is endothelial dysfunction reversible? Am $\mathcal{F}$ Cardiol 1995;76:117A-21A.

40 John S, Schlaich M, Langenfeld M, et al. Increased bioavailability of nitric oxide after lipid-lowering therapy in hypercholesterolaemic patients: a randomized, placebocholesterolaemic patients: a randomized, placebo-
controlled, double-blind study. Circulation 1998;98:211contro

41 van Boven AJ, Jukema JW, Zwinderman $\mathrm{AH}$, et al. Reduction of transient myocardial ischaemia with pravastatin in addition to the conventional treatment in patients with angina pectoris. Circulation 1996;94:1503-5.

42 Andrews TC, Raby K, Barry J, et al. Effect of cholesterol reduction on myocardial ischemia in patients with coronary disease. Circulation 1997;95:324-8.

43 Buchwald H, Varco RL, Matts JP, et al. Effect of partial ileal bypass on mortality and morbidity from coronary heart disease in patients with hypercholesterolaemia. Report of the program on the surgical control of the hyperlipemias (POSCH). N Engl f Med 1990;323:946-55.

44 Brown G, Albers JJ, Fischer LD, et al. Regression of coronary artery disease as a result of intensive lipid lowering therapy in men with high levels of apolipoprotein B. N Eng $\mathcal{F}$ Med 1990;323:1289-98.

45 Watts GF, Lewis B, Brunt JNH, et al. Effects on coronary artery disease of lipid-lowering diet, or diet plus cholestyramine, in the St Thomas' atherosclerosis regression study (STARS). Lancet 1992;339:563-9.

46 Williams JK, Sukhova GK, Herrington DM, et al. Pravastatin has cholesterol-lowering independent effects on the artery wall of atherosclerotic monkeys. $\mathcal{f} \mathrm{Am}$ Coll Cardio 1998;31:684-91.
47 Tsuda Y, Satoh K, Kitadai M, et al. Effects of pravastatin sodium and simvastatin on plasma fibrinogen level and blood rheology in type II hyperlipoproteinaemia. Atherosclerosis 1996;112:225-33.

48 Lacoste L, Lam JYT, Hung J, et al. Hyperlipidemia and coronary disease: correction of the increased thrombogenic potential with cholesterol reduction. Circulation 1995;92: 3172-7.

49 Rosenson RS, Tangney CC. Beneficial effects of statins. Lancet 1996;348:1583.

50 Hoffman R, Brook GJ, Aviram M. Hypolipidemic drugs reduce lipoprotein susceptibility to undergo lipid peroxidation: in vitro and in vivo studies. Atherosclerosis 1992;93:105-13

51 Chen L, Haught WH, Yang B. Preservation of endogenous antioxidant activity and inhibition of lipid peroxidation as common mechanisms of antiatheroscerotic effects of vitamin E, lovastatin and amlodipine. $7 \mathrm{Am}$ Coll Cardiol 1997;30:569-75.

52 Corsini A, Bernini F, Quarato P, et al. Non-lipid-related effects of 3-hydroxy-3-methylglutaryl coenzyme A reductase inhibitors. Cardiology 1996;87:458-68.

53 Adams MR, Jessup W, Celermajer DS. Cigarette smoking is associated with increased monocyte adhesion to endotheial cells: reversibility with oral L-arginine but not vitamin C. F Am Coll Cardiol 1997;29:491-7.

54 Pech-Amsellen MA, Myara I, Storogenko M, et al. Enhanced modifications of low-density lipoproteins (LDL) by endothelial cells from smokers: a possible mechanism of smoking related atherosclerosis. Cardiovasc Res 1996;31: 975-83.

55 Zeiher AM, Schachinger V, Minners J. Long-term cigarette smoking impairs endothelium-dependent coronary arterial vasodilator function. Circulation 1995;92:1094-100.

56 Galis ZS, Asanuma K, Godin D, et al. N-acetyl-cysteine decreases the matrix-degrading capacity of macrophagederived foam cells: new target for antioxidant therapy? Circulation 1998;97:2445-53.

57 Muhlestein JB, Anderson JL, Hammond EH, et al. Infection with Chlamydia pneumoniae accelerates the development of atherosclerosis and treatment with azithromycin prevents it in a rabbit model. Circulation 1998;97:633-6.

58 Gurfinkel E, Bozovich G, Darcoca A, et al. Randomised trial of roxithromycin in non-Q-wave coronary syndromes: ROXIS pilot study. Lancet 1997;350:404-7. 